\title{
Organizing to Implement Technology in the NASA Science Organization
}

\author{
Robert Bauer \\ National Aeronautics and Space Administration \\ Earth-Sun System Technology Office \\ Greenbelt, MD 20771 \\ 301-286-1628 \\ Robert.Bauer@nasa.gov
}

\author{
Michael Pasciuto \\ National Aeronautics and Space Administration \\ Earth-Sun System Technology Office \\ Greenbelt, MD 20771 \\ 301-286-0006 \\ Michael.Pasciuto@nasa.gov
}

\begin{abstract}
As part of a NASA reorganization to support the new Vision for Space Exploration, a number of space and Earth science activities were combined into a single organization. This merger provided an opportunity to review and revise technology development within the new entity. While this process has yet to be finalized, an overview $^{1,2}$ of some of the options and considerations is provided. Examples from one portion of the new entity, the Earth-Sun System Technology program, are used as illustrations.
\end{abstract}

\section{TABLE OF CONTENTS}

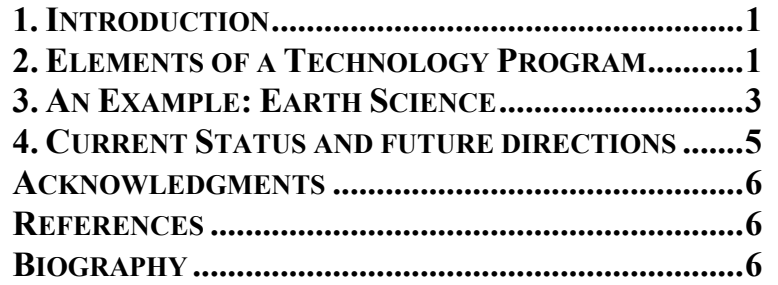

\section{INTRODUCTION}

Since August 2004, NASA has been involved in a reorganization to support the new Vision for Space Exploration [1]. As part of this reorganization, a number of space and Earth science activities were combined into a single organization, known as the Science Mission Directorate (SMD). This merger provided an opportunity to review and revise technology development within the new science entity. While this process is yet to be finalized, an overview of considerations is provided.

Technology is a key activity to enhance and enable science in the new organization. The purpose of this brief paper is not to prescribe precise details of organizational entities, but to identify a set of guiding principles for evaluating technology activities, and to set up a framework for working out details as relationships and processes within the new organization mature.

\footnotetext{
${ }^{1}$ U.S. Government work not protected by U.S. copyright.

${ }^{2}$ IEEEAC Panel 14.08, paper \#3, Version 2, Updated January 31, 2005
}

\section{Guiding Principles}

The following were considered guiding principles for evaluation of options in the transition process:

1) Do no harm. When considering what changes to implement, identify problem areas and focus attention on solutions; do not change what currently works unless there are clear and substantial benefits in making a change.

2) Technology is not an end in itself. The primary customers for technology in the new science organization are the three science divisions (Earth-Sun System, Solar System, and Universe) within the new directorate.

3) One size or approach may not fit all. No organizational structure should be established which introduces new or additional layers or boundaries that buffer the science customers from the technology developers.

These principles are used to evaluate options in the next two sections. It should be noted that both of the former Space and Earth Science entities had successful technology programs. The former Space Science Enterprise developed technology in a focused manner within the Science Divisions/Themes. The former Earth Science Enterprise developed technology through a centralized Earth Science Technology Office (ESTO). Both carried out technology planning, development, and coordination activities discussed in the next section.

\section{Elements of a Technology Program}

\section{A. Planning and Strategy}

Technology cannot be an end in itself - unless it is driven by the science needs of the NASA community, it may rapidly become "sandbox" activity of minimal relevance.

This effort requires studies, draws upon existing plans and roadmaps, and requires constant interaction with and feedback from the sponsors and the science community. Some of these activities are at the highest NASA levels, 
while others will be more specific to a particular science discipline. So how might this apply in the new SMD technology structure?

Several technology functions supporting the missions of the SMD science divisions are best accomplished at a high level within the Directorate. Organizationally, a strategy and policy function should come under the auspices of the Assistant Associate Administrator for Technology (AAA-T) that reports directly to the AA's office. The AAA-T represents SMD technology interests to the NASA Advanced Planning Integration Office (APIO) and other Agency planning activities, and acts with delegated authority of the SMD Associate Administrator with respect to advanced technology.

The AAA-T may coordinate policies and practices across the Directorate by chairing a Technology Management Board. A primary goal of this Board is to ensure communication and information exchange across the Science Divisions. However, the technologists who understand the science needs and requirements best are the Division/Theme technologists. To avoid the danger of having technology become and end unto itself, the actual technology implementation should stay within each SMD Science Division, close to the science it is serving. The Other coordination functions include interfacing with advanced technology in other Agencies, and within NASA, the Exploration Systems Mission Directorate (ESMD). New technology programs/initiatives would be advocated at this level, and it would include an aspect of the Directorate's Education/public and professional outreach (E/PO) and advocacy function as it relates to technology.

\section{B. Implementation}

There are several options for carrying out technology development within SMD. These should be applied as they are most appropriate to support the science. As stated in the Introduction, the former Space Science Enterprise developed technology in a focused manner within the Science Divisions/Themes. The former Earth Science Enterprise developed technology through a centralized Earth Science Technology Office (ESTO).

There is a fundamental underlying reason for the centralized approach to Earth Science technology development. To understand the complex nature of the processes which govern our planet, data and information from many models and sensors must be combined in a systematic fashion. Even though Earth Science research is described in terms of six "focus areas", the approach to Earth Science research was to treat the Earth as an integrated system ("Earth System Science" (Figure 1)). Hence the technology program was also designed to integrate across focus areas to support a systematic approach to enabling the new science measurements. Both the former Code $\mathrm{S}$ Theme/Division approach and the ESTO Earth System
Science approach were, and still are, science-driven. Hence, all three divisions within the SMD use a sciencedriven technology development paradigm, but implement their technology program in a manner to best support their science. One size or approach may not fit all. Each science division best knows its needs, schedule, and the resources that it can dedicate to technology.

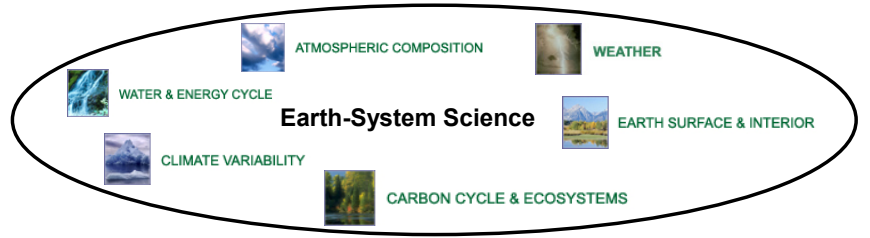

Figure 1 - The Six Focus Areas Which Comprise Earth System Science

\section{Coordination}

In the current budget-constrained environment, some technologies may address a number of science problems. To avoid duplication, some sort of cross-organization communication is essential. But to minimize additional organizational structure (guiding principle \#3), this could take the form of a technology working group or committee rather than a specific new cross-organizational entity. In fact, this type of coordination is already occurring within SMD without any formal structure. Some general observations will be made here about technology coordination, and then specific examples will be provided of actual cross-SMD technology collaborations which are already in progress will be discussed in Section 4.

Among the SMD science divisions there may be a sub-set of technologies that can be mutually beneficial. Initially, technology requirements development will be implemented within each division which has the best understanding of their science needs. As the directorate organization matures, technology research conducted within the EarthSun System, Solar System, and Universe Divisions would be reviewed for common requirements and technology needs. Those could form the basis for a collaborative technology development program to serve the entire SMD. This collaborative technology effort would require funding. This budget would be determined at the Science Division level. Once again, the customers for technology within SMD are the Science Divisions. They should "vote with their wallets". The intent would be for these broad-based and exploratory technologies to be managed separately from individual science mission projects with their near-term budget priorities and liens. This would help to ensure that the typical longer duration development time be permitted. After risk is sufficiently retired, a Division may (or may not) pick-up task maturation as a mission-focused technology. 
This same analog can be applied to technology validation. Validation can be sub-orbital (e.g. aircraft, balloon, uncrewed aerial vehicle (UAV)) or space-borne. For example, the current Earth-Sun System Division's UAV-synthetic aperture radar (SAR) validation project might be of interest to the Solar System Division if a P-band component were added to the radar. Space flight validation can be either a New Millennium-like program or collaboration with another NASA Directorate or even an external agency. There is currently collaboration among the Air Force Flight Research Laboratory's Space Vehicles Directorate, NASA's Space Flight Directorate and SMD's Earth-Sun System Division to flight validate a software-defined transceiver on an Air Force Research Laboratory (AFRL) mission. In this instance, each NASA entity and AFRL reviewed their requirements (and budgets) and an interagency Memorandum of Agreement was established for the collaboration. There should be no one size fits all validation approach. Each science division should decide what approach best fits their requirements.

\section{An EXAMPLE: EARTH SCIENCE}

Section 2 provided an overview of the kinds of activities a technology organization needs to perform: planning, strategy, implementation, and coordination. This section shows how one of the technology programs in the science directorate - ESTO - accomplished those activities. After a brief history of how ESTO was formed, the section describes how it plans and implements technology activities, and gives an overview of progress and successes.

\section{A. Background and Guiding Principles}

In June of 1997, the Earth Science Biennial Review recommended that future Earth Science missions be implemented with shorter development time and using the best suitable technology. Responding to these recommendations, NASA's Earth Science Enterprise developed a plan which:

- included the establishment of a flexible, science-driven technology strategy,

- would develop very specific technologies via a competitive selection process and

- would provide a broad portfolio of emerging technologies for infusion into a range of Earth Science missions.

The Earth Science Technology Program was established to meet these challenges, and the Earth Science Technology Office (ESTO) was created in March 1998 as the responsible organization. Since inception, technologies developed through the program -- a portfolio of more than 400 investments at over 70 institutions nationwide, including advancements in sensors, instruments, communication systems, and computer modeling -- have already been incorporated into numerous Earth and space science missions as well as commercial applications

A number of principles have contributed to the success of ESTO activities. Organized into general areas within the research development process, they include:

Planning and Setting Directions. Each solicitation identifies specific science-driven areas of emphasis and criteria for evaluation. The science needs are taken from Science Roadmaps as well as being drawn form open community workshops. This process is facilitated by use of the Earth Science Technology Integrated Planning System (ESTIPS). The Earth Science Technology Integrated Planning System can be found at http://estips.gsfc.nasa.gov. Figure 2 shows the ESTIPS home page. This system was developed several years ago to support the existing NASA Earth Science Enterprise in translating science questions and measurements into new measurement scenarios and their associated technology requirements. Before the release of any solicitation, a final coordination is made with each NASA science focus area lead. This narrows the scope of the solicitation and by reducing the potential number of proposals submitted it also reduces the overhead associated with writing and reviewing large numbers of proposals. A very comprehensive explanation of "Deriving Technology Needs From Measurement Strategies" may be found in Stabnow [2].

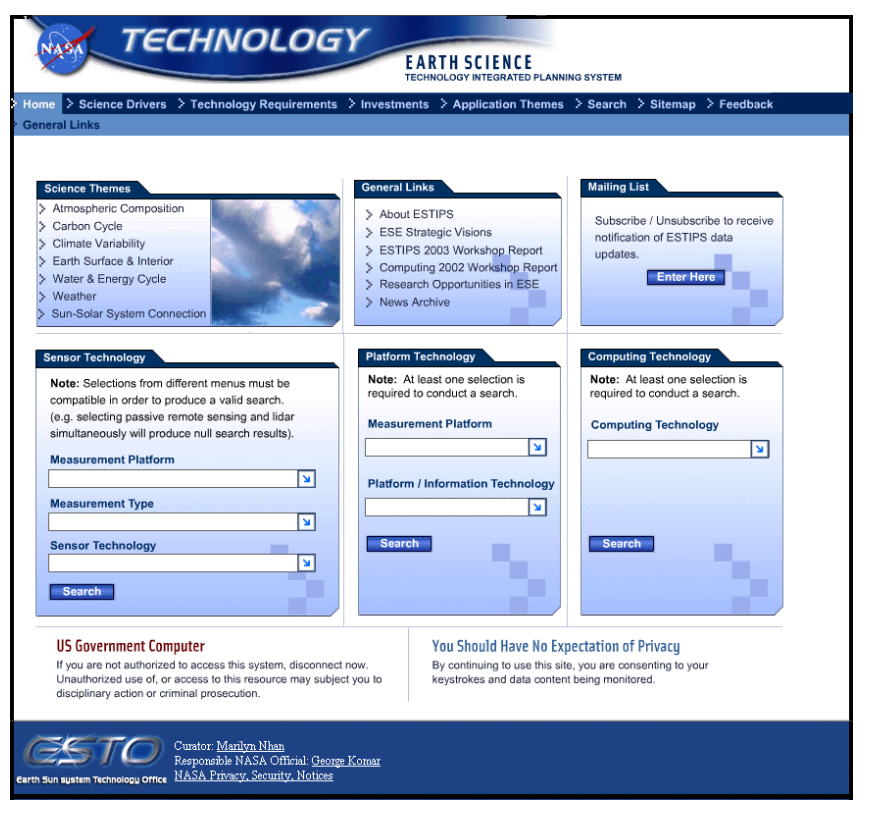

Figure 2 - The Earth Science Technology Integrated Planning System

- Implementation: Soliciting Ideas. The program is based upon competitive, peer-reviewed proposals; this allows selection of best-of-class technology investments. 
Technologies are infused into a mission by competitive selection of science investigators or mission managers, not the Earth Science Technology Program. The awards are for up to three years, but solicitations are issued approximately every two years; this overlapping of award periods avoids the "dry spell' phenomenon, where researchers leave the research arena when there are no new opportunities. Finally, the programs fund at requested levels; if an award makes it through the review and approval process, further budget negotiations are detrimental to partnering and leveraging opportunities established in the proposal.

- Implementation: Guiding Progress. One technique which the program has used very successfully is distributed management: ESTO awards are administered using geographically distributed organization in which local NASA Centers assume responsibility for key aspects of the program; since many missions and investigations are at these NASA centers, the distributed organization allows technology to develop closest to potential users. The Earth Science Technology program also includes periodic independent reviews to evaluate progress and potential. The Aerospace Corporation, a Federally Funded Research and Development Center (FFRDC), participates in annual reviews of each award, and provides a report on status, problems, and possible directions. Each awardee has a set of metrics against which to measure progress, and the program as a whole also has specific criteria against which it is evaluated. For example, one of the Performance Targets and Related Indicators for the Earth Science Enterprise is "Annually advance at least $25 \%$ of funded technology developments one TRL".

- Coordination: Encouraging Utilization. Finding an eventual home for each technology is included throughout the research process. While the research award is in progress, it is monitored by the distributed Earth Science Technology organization, at the particular NASA center where potential missions and investigations are focused. The program also has frequent interaction options built into it, including an annual Earth-Sun System Technology Conference (ESTC) which rotates among NASA locations. The competitively-selected principal investigators discuss their own research. This exposes the technology research to their peers in academia, industry and other NASA centers. No technology is ever "forced" on a science mission or campaign. Technology is infused by merit alone.

\section{B. Program Activities and Progress to Date}

The ESTO program includes four distinct but related elements:

- Advanced Technology Initiatives (ATI) - provides for concept studies and development of component (Advanced Component Technology Program) and subsystems technologies for instruments and platforms.
Instrument Incubator Program (IIP) - provides new instrument and measurement techniques including lab development and airborne validation.

- Advanced Information Systems Technologies (AIST) provides innovative on-orbit and ground capabilities for the communication, processing, and management of remotely sensed data and the efficient generation of information.

- Computational Technologies (CT) - provides techniques and systems which enable high performance throughput, archiving, data manipulation, and visualization of very large, highly distributed remotely sensed data sets consistent with modeling needs.

ESTO is also the lead for a NASA-wide targeted research activity known as the Laser Risk Reduction Program (LRRP). This program was established to address recommendations to NASA by an external committee to understand and reduce the risk of the space application and use of laser technology.

These program elements span the Technology Readiness Level (TRL) between Basic Principles (TRL 1) and Flight Qualified Prototypes (TRL 6-7).

There were 138 awards from these ESTO programs which were active during fiscal years 2003 and 2004 alone. These awards were to academia, industry, federal labs and NASA centers. This distribution of awards is shown in Figure 3.

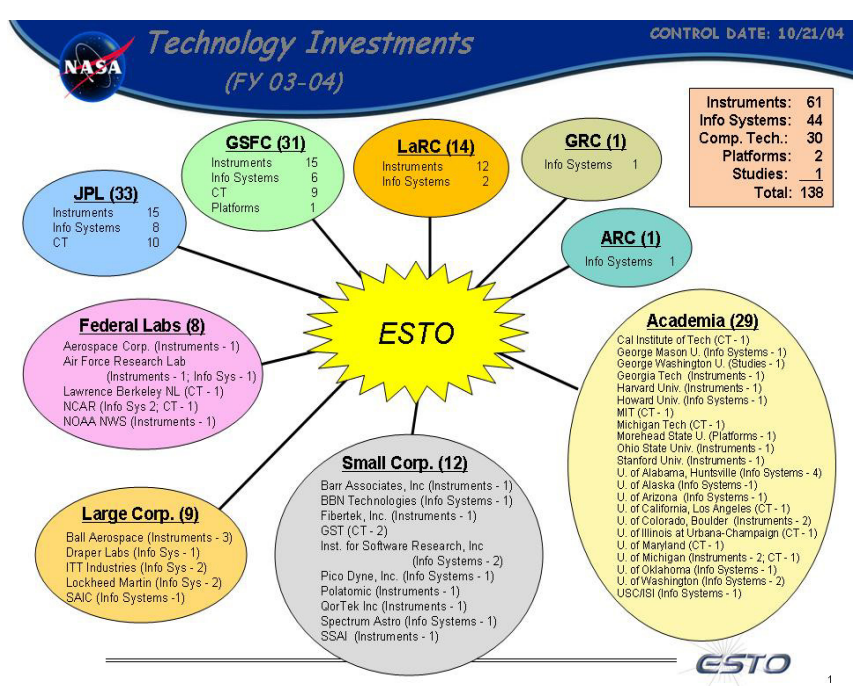

Figure 3 Distribution of Active Technology Projects by Organization for FY 2003-2004

The list of program accomplishments is substantial. ESTO technology is a part of every proposed NASA Earth Science mission after Cloudsat. The vast majority of research activities have made technical progress; a majority of ESTO awards have been incorporated into some sort of system or 
application. The program has also had notable educational implications: a number of student researchers, from high school through $\mathrm{PhD}$, have contributed to these Earth Science technology improvements, and innovations developed through this program have contributed toward advanced degrees for several students. Technology awards have progressed using to a simple metric - advancement in Technology Readiness Level. Of all completed projects, $77 \%$ have advanced at least one TRL level, and many advanced more than one TRL level for each year of the project.

\section{Current Status and Future Directions}

\section{A. Current Status}

As mentioned in the Section 2, changes are already occurring and collaborations taking place within SMD. ESTO is now the technology office for the Earth-Sun System Division (ESSD). This division combines the former Earth System Science (ESS) Research and the SunEarth Connection (SEC) science programs. Both the ESS and SEC had successful technology development programs, coupled directly to their science customers. As suggested in the Introduction, there are Guiding Principles which can be applied to the evolving structure of technology development in the SMD. Let us review those in the context of the recently described ESTO functions. In the vein of do no harm, the new ESSD technology program has kept the respective science-focused linkages and road mapping activities. However, technologists from the former SEC (now Sun Solar System Connection (SSSC)) and ESTO are now participating jointly in these activities to find synergies. An initial review of each technology program has revealed some common needs such as: ultra low power radiation tolerant electronics, large structures, increased onboard autonomy and computation.

As part of ESSD's upcoming Advanced Component Technology (ACT) NASA Research Announcement (NRA) [3], technology requirements have been included for both programs. Also, the SSSC technology requirements will be hosted in ESTIPS. In the past both ESTO and the SSSC technologist have utilized detailed technical trade studies to narrow down competing technology options. An example was that there were six competing technology approaches to making a cold land process measurement. There was not enough funding to try to develop all of these options. Under ESTO leadership, a team was formed of scientists and technologist from academia and NASA. The review resulted in cutting the trade-space to only two preferred approaches. This saved both time and funding. ESTO will work with the SSSC lead technologist to perform studies to refine the results of the current SSSC technology roadmap activities.
Prior ESTO ACT technologies have also been utilized by other SMD divisions. For example, ESTO transmit/receive $(\mathrm{T} / \mathrm{R})$ modules for precipitation detection have been adopted and modified by the Mars Focused Technology Program to become the $\mathrm{T} / \mathrm{R}$ modules for the Mars 2009 hazard avoidance radar. ESTO-developed digital receiver chips have been adopted by the NASA KA-band transition program and installed at the White Sands ground station. These chips have been technology-transferred to industry and are now a commercial product which is being considered for the Solar Dynamic Observatory ground station. Anticipating that there may be certain other synergies which could come from the current ESTO ACT procurement, we have given copies of the drafts (and final) NRA technical requirements to the other Division and Theme technologists within the SMD. We have also invited them to participate in the reviews of the ACT proposals. We anticipate that this type of pro-active collaborative approach will enable cost saving and perhaps even more awards to be made.

Another area in which we are actively collaborating with our colleagues in the SMD is availing them of the opportunity to participate in the annual Earth-Sun System Technology Conference (ESTC). The ESTC is held annually and brings together principal investigators who have active ESSD awards. The former SEC technology program will have a session of papers. We are making the conference available to all SMD technologist as well as their principal investigators.

We have also pursued collaboration with the former Space Science Advanced Information Systems Research Program (AISRP). ESTO staff will participate in the AISRP annual meeting this April. ESTO and AISRP staff have in the past already participated in the review of each others proposals. Since information systems is such a cross-cutting technology area, we anticipate that our next ESSD Advanced Information System Technology (AIST) NRA we will again solicit inputs from all of our colleagues in the other SMD technology programs. This pro-active approach will lead to synergies where appropriate and only to the level wherein the other divisions decide it is appropriate to collaborate with their funds.

As one can see from these few examples, without any formal structure, and in less than a year, technology coordination and collaboration is beginning to thrive in the SMD. No organizational structure should be established which introduces new or additional layers or boundaries that inhibit this type of technology collaboration. 


\section{REFERENCES}

\section{B. Future Directions}

In the next several months, a number of NASA-wide activities will affect technology:

- Strategic roadmapping activities will set directions and priorities in the dozen areas with which NASA is involved;

- Technology capabilities evaluation activities will turn those strategic roadmaps into a set of key priorities;

- The science organization will release an umbrella solicitation for research (ROSES - 1/28/05) [3];

- The science organization will begin to set up a framework and organization for handling technology in the future.

Although a number of possible organizational arrangements are possible, a strong connection to science, and an open and unconstrained interaction within the SMD technology community, will be key attributes of a future organization. A technology organization cannot be an end unto itself; its sole function is to enable science.

\section{ACKNOWLEDGMENTS}

The authors would like to thank their fellow technologists in the NASA Science Mission Directorate and our ESTO Associates at the NASA Centers. We look at this as an opportunity to enhance all of our technology programs.
[1] The Vision for Space Exploration. http://www1.nasa.gov/pdf/55583main_vision_space_expl oration2.pdf.

[2] Willam Stabnow, "Deriving Technology Needs from Measurement Strategies," 2005 IEEE Aerospace Conference, Big Sky, Montana: Panel 14.08, Paper \#2.

[3] The ESSD ACT procurement will be an element in the SMD "Research Opportunity for Space and Earth Science" (ROSES) $2005 \quad$ NRA. (http://www.fedgrants.gov/Applicants/NASA/HQ/OPHQ DC/NNH05ZSD001N/listing.html)

\section{BIOGRAPHY}

Robert Bauer is a technology development manager in the

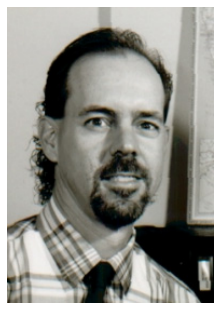
NASA's Earth- Sun System Technology Office (ESTO). He is the office's liaison with NASA Headquarters where he is responsible for coordinating technology development activities in support of Earth science between Headquarters and ESTO. Most recently he has been involved with the planning of technology transformation with the new Science Mission Directorate. He came to ESTO in 2002 after being project manager of the Advanced Communications Technology Satellite (ACTS) at NASA's Glenn Research Center. He received a Bachelor's in Electrical Engineering and a Masters in Business Administration from Cleveland State University.

Michael Pasciuto is the Lead for the Planning Group in the

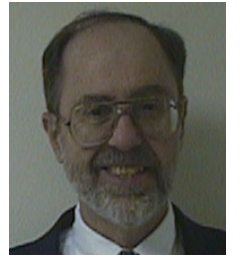
Earth-Sun System Technology Office. His group is responsible for requirements development and capture, studies and analyses, technology partnering, outreach and the Advanced Component Technology Program. He previously worked in the aerospace industry where he was responsible technology requirements of various DoD and space missions including the Hubble Space Telescope science and operations ground system. He came to NASA Headquarters in 1988 where he worked on Space Station Freedom and Office of Space Science information and communications systems. He later became the Hubble Space Telescope manager for Servicing Mission 2. He came to the Goddard Space Flight Center in 1996 to work in the Earth System Science Program Office and then in 1999 he joined the Earth Science Technology Office. He has a Bachelor of Science degree in Physics and Mathematics from St Johns University in NY, and a Master 
of Science Degree in Physics from the Polytechnic Institute of Brooklyn, NY. 